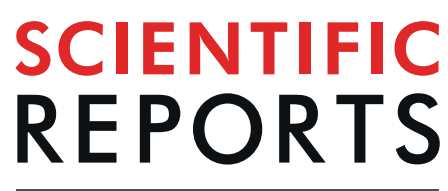

natureresearch

\title{
OPEN Fine-tuning the expression of target genes using a DDI2 promoter gene switch in budding yeast
}

Received: 16 November 2018

Accepted: 21 May 2019

Published online: 29 August 2019

\author{
Yong Wang ${ }^{1,2}$, Kaining Zhang ${ }^{1,2}$, Hanfei $\mathrm{Li}^{1,2}, \mathrm{Xin} \mathrm{Xu}^{1}$, Huijun Xue ${ }^{1}$, Pingping Wang ${ }^{3}$ \& \\ YuV. Fu' ${ }^{1,2}$
}

Tuned gene expression is crucial to the proper growth and response to the environmental changes of an organism. To enable tunable gene expression as designed is desirable in both scientific research and industrial application. Here, we introduce a novel promoter switching method based on the DDI2 promoter $\left(\mathrm{P}_{D D 12}\right)$ that can fine tune the expression of target genes. We constructed a recyclable cassette $\left(\mathrm{P}_{D D 12}-U R A 3-\mathrm{P}_{D D 12}\right)$ and integrated it upstream of yeast target genes to replace the native promoters by $D D I 2$ promoter without introducing any junk sequence. We found that the presence or absence of cyanamide as an inducer could turn on or off the expression of target genes. In addition, we showed that $P_{D D 12}$ could act as a gene switch to linearly regulate the expression levels of target genes in vivo. We switched the original promoters of RAD18, TUP1, and CDC6 with $\mathrm{P}_{D D 12}$ as a proof-of-concept.

For each cell, the transcriptional programs are modified to maintain specific intracellular conditions to ensure optimal growth and function ${ }^{1,2}$. When environmental conditions change suddenly, the ability of cells to rapidly adjust genome expression is critical for competitive fitness and cell survival ${ }^{3,4}$. Unicellular organisms like Saccharomyces cerevisiae have evolved autonomous mechanisms in response to changes in the environment during organismal development ${ }^{2,5}$. Among them, the promoter is the most basic and the most important tool to control gene expression programs. Various strengths of constitutive and inducible promoters provide a broad range of genetic control in S. cerevisiae $e^{6,7}$.

Constitutive promoters maintain relatively stable expression levels and do not require inducers or repressors $^{8,9}$. The widely used constitutive promoters in budding yeast regulate genes involved in the glycolytic pathway, such as the promoters of alcohol dehydrogenase $1\left(\mathrm{P}_{A D H 1}\right)^{10}$ and phosphoglycerate kinase $\left(\mathrm{P}_{P G K}\right)^{11}$. Inducible promoters are more suitable than constitutive promoters for regulating gene expression in response to stimuli, which may be desirable for the fine-tuning purpose. The use of inducible promoters is limited by the strength of the promoter's response to the inducer, the leak degree of the expression controlled by the promoter, and the cost of induction ${ }^{9}$. In yeast, the most commonly used tightly-inducible promoters are $\mathrm{P}_{\text {GAL1 }}{ }^{12}, \mathrm{P}_{G A L 10}$, and $\mathrm{P}_{\text {GAL7 }}{ }^{13,14}$, which are induced by galactose and strongly repressed in glucose medium ${ }^{15}$. Yeast cells prefer glucose and fructose as carbon sources, thus when $\mathrm{P}_{G A L 1}$ is used to induce target gene expression, the original carbon source must be replaced by galactose. The process of changing medium is difficult and galactose is considered too expensive for use in large-scale cultures ${ }^{8} . \mathrm{P}_{C U P 1}$ is another commonly used inducible promoter in budding yeast, which is activated by $\mathrm{Cu}^{2+, 16}$. Compared to $\mathrm{P}_{G A L 1}$ promoter, the $\mathrm{P}_{C U P 1}$ promoter displays rather high basal level expression in the absence of $\mathrm{Cu}^{2+17,18}$. Moreover, Cooper could be enriched inside the cells that make a serious impact on both cellular structures and metabolisms. The tetracycline regulatory system (Tet-on and Tet-off), which is originally from bacteria, has been widely used to regulate gene expression in eukaryotes ${ }^{19}$. In eukaryotic cells, this system has been applied to control the RNA polymerase III-driven transcription of eukaryotic tRNA genes ${ }^{20,21}$. However, this system requires the introduction of heterogeneous regulatory proteins into host cells ${ }^{19,22}$. Therefore, simple and efficient inducible promoters are needed in S. cerevisiae.

The DDI2 and DDI3 genes were reported to display the highest induction ( $>100$-fold) in yeast cells after treatment with the DNA-damaging agent methyl methanesulfonate (MMS) ${ }^{23}$. DDI2 and DDI3 are two identical genes with exactly the same ORF sequences and only one nucleotide difference in their promoters ${ }^{24}$, but they are

${ }^{1}$ State Key Laboratory of Microbial Resources, Institute of Microbiology, Chinese Academy of Sciences, Beijing, 100101, China. 'Savaid Medical School, University of Chinese Academy of Sciences, Beijing, 100101, China. ${ }^{3}$ Qingdao Baihuizhiye Biotech Co.Ltd, Qingdao, 266109, China. Yong Wang and Kaining Zhang contributed equally. Correspondence and requests for materials should be addressed toY.V.F. (email: fuyu@im.ac.cn) 
a

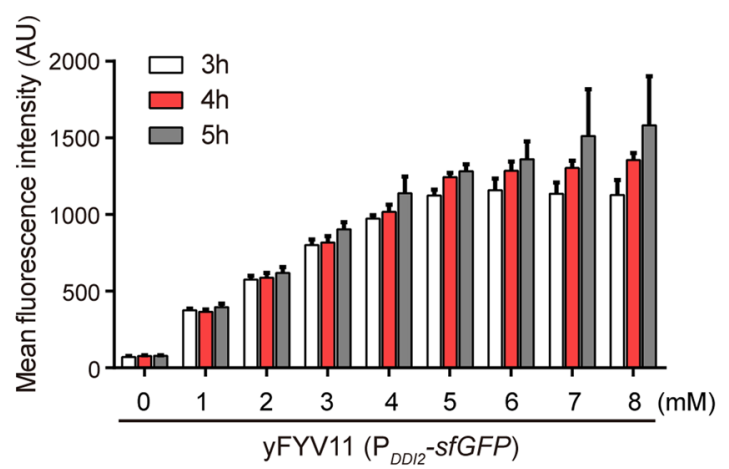

b

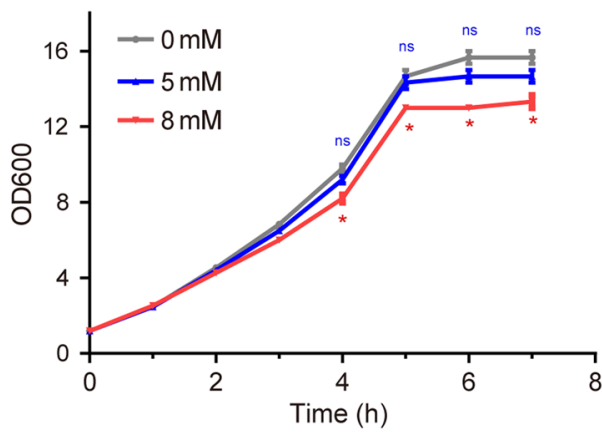

Figure 1. Optimal DDI2 promoter induction time and concentration analysis. (a) Mean fluorescence intensity of the yFYV11 ( $\left.\mathrm{P}_{D D I 2}-s f G F P\right)$ strain under the control of $\mathrm{P}_{D D I 2}$ at different induction time points $(3-5 \mathrm{~h})$ and different cyanamide concentrations $(0-8 \mathrm{mM})$ observed by flow cytometry. (b) Effect of cyanamide on the growth rate of yeast cells. After adding different concentrations of cyanamide $(0,5$, and $8 \mathrm{mM})$, OD600 values were measured every hour for $7 \mathrm{~h}$. The $5 \mathrm{mM}$ cyanamide hardly affected the growth rate of the yeast cells (ns: not significant), however, $8 \mathrm{mM}$ cyanamide affected BY4741 cell growth $(* * p<0.01)$. (t test, $\mathrm{n}=3, * \mathrm{p}<0.05$, $* * \mathrm{p}<0.01, * * * \mathrm{p}<0.001)$.

located on different chromosomes. Protein sequence analysis and experimental verification revealed that DDI2/3 encode a cyanamide hydratase in S. cerevisiae ${ }^{25-27}$. Meanwhile, it has been reported that cyanamide can highly induce the expression of $D D I 2 / 3$ as $\mathrm{MMS}^{24,28}$. Cyanamide is a clean fertilizer because in the air it can be converted naturally into ammonia and carbon dioxide ${ }^{24}$. Cyanamide is also used as an alcohol deterrent drug ${ }^{29,30}$ and as a raw compound in the pharmaceutical industry to produce guanidine derivatives ${ }^{31}$. Hence, we propose to utilize the DDI2 promoter to develop a novel gene expression regulating system induced by cyanamide.

Here, we used the superfolder green fluorescent protein gene $(s f G F P)$ as a reporter gene to determine the strength and regulation of $\mathrm{P}_{D D I 2}$ compared with three other widely-used promoters $\left(\mathrm{P}_{A D H 1}, \mathrm{P}_{C U P 1} \text { and } \mathrm{P}_{\text {GAL1 }}\right)^{32}$. Quantitative analysis results demonstrated that the induction level of $\mathrm{P}_{D D I 2}$ is strong and leaky level is low. As a proof-of-concept, we replaced the native promoters of three yeast genes (RAD18, TUP1, and CDC6) with $\mathrm{P}_{D D I 2}$, without redundant DNA sequence left. As anticipated, $\mathrm{P}_{D D I 2}$ showed tunable control of the target genes, especially the regulation of the essential gene CDC6 as a yeast safeguard switch. We demonstrated a linear gene expression control on the target gene correlated with the inducing strength, which further facilitates the precise regulation of gene expression in budding yeast.

\section{Results}

Optimal DDI2 promoter induction time and inducer concentration. $\mathrm{P}_{D D I 2}$ is a novel inducible promoter that is efficiently induced by cyanamide ${ }^{24,28}$. We tested whether the strength of $\mathrm{P}_{D D I 2}$ is affected by both the induction time and inducer concentration. To determine the optimal induction time and cyanamide concentration, we established a reporter gene system in which an sfGFP gene is under the control of DDI2 promoter (see Supplementary Fig. S1a). To avoid the potential effect from gene copy number variations a single-copy plasmid YCp (yeast centromere plasmid) was chosen as the backbone vector ${ }^{33}$. We measured the expression of the $\mathrm{P}_{D D I 2}$-sfGFP reporter gene at different induction time points $(3-5 \mathrm{~h})$ and cyanamide concentrations $(0-8 \mathrm{mM})$ by flow cytometry. As shown in Fig. 1a, the mean fluorescence values of $s f G F P$ increase with increasing cyanamide concentrations $(0-8 \mathrm{mM})$ and stabilize when cyanamide concentrations are $5 \mathrm{mM}$ and above. The highest $s f G F P$ expression level is observed after 5-hour induction among 3-5 hour induction time points, which implies that $\mathrm{P}_{D D I 2}$ activity is proportional to the induction time.

It has been reported that cyanamide has modest toxicity to humans ${ }^{34}$. To investigate the effect of cyanamide toxicity on the growth of yeast cells, we generated growth curves for wild-type S. cerevisiae cells grown in YPD medium supplemented with 0,5 and $8 \mathrm{mM}$ cyanamide. Figure $1 \mathrm{~b}$ shows that the growth rate of yeast cells is hardly affected by $5 \mathrm{mM}$ cyanamide, but with $8 \mathrm{mM}$ cyanamide the cell concentration (OD600) is slightly affected and at longer induction time the cell growth rate is lower than at 0 and $5 \mathrm{mM}$ cyanamide concentrations. These data suggest that $\mathrm{P}_{D D I 2}$ is induced efficiently by cyanamide and has high promoter activity and that cyanamide has little effect on cell growth at concentrations equal to or lower than $5 \mathrm{mM}$.

Comparison of the DDI2 promoter with ADH1, CUP1 and GAL1 promoters. We compared the strength and induction process of $\mathrm{P}_{D D I 2}$ with three classic promoters, $\mathrm{P}_{A D H 1}, \mathrm{P}_{C U P 1}$ and $\mathrm{P}_{G A L 1}$. To facilitate the comparisons, we established the same reporter gene system as described above just by replacing DDI2 promoter with three other promoters. The four plasmids carrying $\mathrm{P}_{D D I 2}-s f G F P, \mathrm{P}_{A D H 1}-s f G F P, \mathrm{P}_{C U P 1}-s f G F P$ and $\mathrm{P}_{G A L 1}-s f G F P$ (see Supplementary Fig. S1a-d) were transferred to the S. cerevisiae cells. After the yeast cells were induced by corresponding inducer or water for 3 hours, we irradiated cells with the $488 \mathrm{~nm}$ laser to observe sfGFP signal by fluorescence microscopy. As shown in Fig. 2a, the fluorescence intensity of yFYV11 ( $\left.\mathrm{P}_{D D I 2-s f G P}\right)$ cells is much greater than that of yFYV9 $\left(\mathrm{P}_{A D H 1-s f G F P}\right)$ cells. Comparing the three inducible promoters, the yFYV11 $\left(\mathrm{P}_{D D I 2-s f G F P}\right)$ cells show higher fluorescence intensity than both yFYV10 $\left(\mathrm{P}_{\text {GAL1-sfGFP }}\right)$ and yFYV17 $\left(\mathrm{P}_{\text {CUP1-sfGFP }}\right)$ cells, (Fig. 2a). 
a

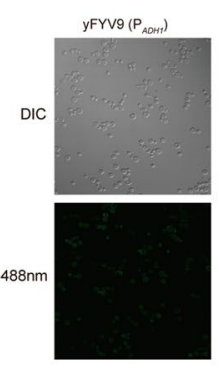

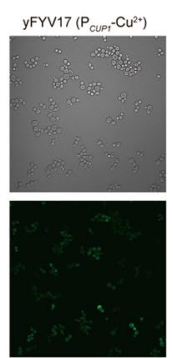
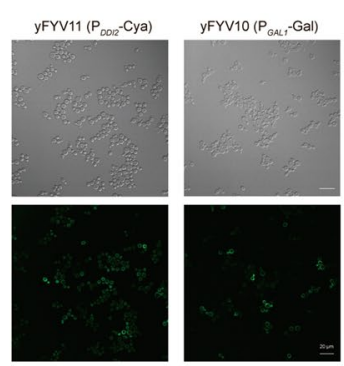

b

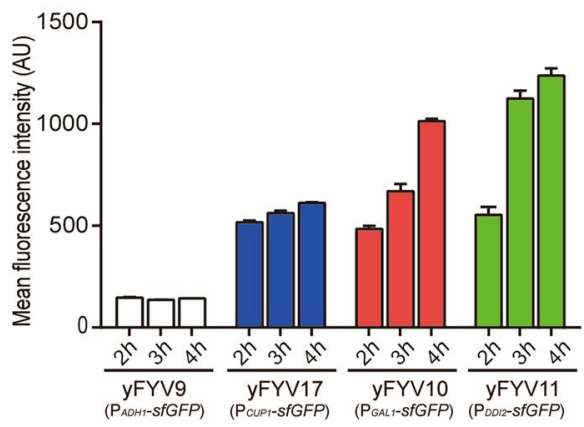

Figure 2. $s f G F P$ expression under the control of four promoters. (a) Comparison of yFYV9 $\left(\mathrm{P}_{A D H 1}-s f G F P\right)$, yFYV17 ( $\left.\mathrm{P}_{C U P 1}-s f G F P\right)$, yFYV10 $\left(\mathrm{P}_{G A L 1}-s f G F P\right)$, and yFYV11 $\left(\mathrm{P}_{D D I 2}-s f G F P\right)$ fluorescence intensities under the control of $\mathrm{P}_{A D H 1}, \mathrm{P}_{C U P 1}, \mathrm{P}_{G A L 1}$, or $\mathrm{P}_{D D I 2}$. The yFYV11 ( $\left.\mathrm{P}_{D D I 2}-s f G F P\right)$ strain was induced by $5 \mathrm{mM}$ cyanamide (Cya) for $3 \mathrm{~h}$, the yFYV10 $\left(\mathrm{P}_{G A L 1}-s f G F P\right)$ strain was induced in galactose $(\mathrm{Gal})$ medium for $3 \mathrm{~h}$ and the yFYV17 $\left(\mathrm{P}_{C^{\prime}}\right)^{-}$ $s f G F P)$ strain was induced in $0.5 \mathrm{mM} \mathrm{Cu}^{2+}$ medium for $3 \mathrm{~h}$. (b) Mean fluorescence changes of yFYV9 $\left(\mathrm{P}_{A D H 1^{-}}\right.$ $s f G F P), y F Y V 17\left(\mathrm{P}_{C U P 1}-s f G F P\right)$ and yFYV10 $\left(\mathrm{P}_{G A L 1}-s f G F P\right)$ strains at different time points by flow cytometry. The yFYV10 $\left(\mathrm{P}_{G A L 1}-s f G F P\right)$ strain was induced in galactose $(\mathrm{Gal})$ medium for $2-4 \mathrm{~h}$ and the yFYV17 $\left(\mathrm{P}_{C}\right.$ IP1 $^{-}$ $s f G F P)$ strain was induced in the medium containing $0.5 \mathrm{mM} \mathrm{CuSO}_{4}$ for $2-4 \mathrm{~h}$.

To get quantitive data to confirm these observations, we measured the expression of sfGFP using flow cytometry in different conditions. For yFYV17 ( $\left.\mathrm{P}_{C U P 1}-s f G F P\right)$ cells, a final concentration of $0.5 \mathrm{mM} \mathrm{CuSO}_{4}$ was applied for induction according to previous study ${ }^{35}$, then the mean fluorescence values were measured at different time points. yFYV10 ( $\left.\mathrm{P}_{G A L 1}-s f G F P\right)$ cells were grown to log phase in glucose medium, then washed and incubated in raffinose medium for 3 hours, which does not repress or induce transcription of the GAL1 promoter ${ }^{36}$, prior to inducing in the galactose medium for 2-4 hours as described in the previous reports ${ }^{37-39}$. The yFYV11 $\left(\mathrm{P}_{D D I 2}-s f G F P\right)$ cells were directly induced by $5 \mathrm{mM}$ cyanamide for $2-4$ hours.

As it is expected, the mean fluorescence values for yFYV9 $\left(\mathrm{P}_{A D H 1}-s f G F P\right)$ cells are relatively low and stable at different time points (Fig. 2b). At the 4-hour time point, compared the mean fluorescence values of yFYV11 $\left(\mathrm{P}_{D D I 2}-s f G F P\right)$ cells induced with $5 \mathrm{mM}$ cyanamide to that of yFYV9 $\left(\mathrm{P}_{A D H 1}-s f G F P\right)$ cells and yFYV17 $\left(\mathrm{P}_{C U P 1}-s f G F P\right)$ induced by $0.5 \mathrm{mM} \mathrm{CuSO}_{4}, \mathrm{P}_{D D I 2}$ exhibited upwards of an 8.72 -fold and a 2.03 -fold increase in mean fluorescence over $\mathrm{P}_{A D H 1}$ and $\mathrm{P}_{C U P 1}$. After 4-hour induction, the mean fluorescence of $y$ FYV10 $\left(\mathrm{P}_{G A L 1}-s f G F P\right)$ reaches a rather high level. However, the mean fluorescence of $\mathrm{P}_{D D I 2}$ is still 1.23-fold higher than that of $\mathrm{P}_{G A L 1}$ at this time point (Fig. 2b). These results suggest that $\mathrm{P}_{D D I 2}$ has significantly higher activity than $\mathrm{P}_{A D H 1}, \mathrm{P}_{C U P 1}$, and $\mathrm{P}_{G A L 1}$ at the same induction time. Hence, $\mathrm{P}_{D D I 2}$ can be used as a novel promoter with high inducibility.

Switching the native promoters of target genes RAD18 and TUP1 with DDI2 promoter. $\mathrm{P}_{D D I 2}$ can be induced by cyanamide, therefore, it is reasonable that the native promoter of a target gene on its genomic locus could be replaced with $\mathrm{P}_{D D I 2}$ to regulate its expression. As a proof-of-concept, we first replaced the promoters of two target genes (RAD18 and TUP1) with $\mathrm{P}_{D D 12}$. RAD18 plays an important role in DNA damage repair caused by UV or methyl methanesulfonate ${ }^{23,40}$. TUP1 is a general repressor of transcription in yeast, and a tup $1 \Delta$ strain exhibits an obvious clumpy cell morphology phenotype $e^{41,42}$ that can be observed directly.

We used the recyclable cassettes method reported previously ${ }^{37,43}$ to replace the native promoter in the yeast genome by one-step transformation followed by two-step selection. First, the $\mathrm{P}_{D D I 2}-U R A 3-\mathrm{P}_{D D I 2}$ (DUD) cassettes were amplified by PCR to replace the promoter of the target gene by homologous recombination. Secondly, the cassette-integrated strain was obtained using auxotrophic plates (SD-URA). The most important step is that one copy of the $\mathrm{P}_{D D I 2}$ and URA3 deleted cells are selected on 5-FOA plates so that only one $\mathrm{P}_{D D I 2}$ regulating the target gene is obtained without redundant DNA. For more efficient promoter shuffling, the several hundred base pairs flanking the homology arms were generated by overlapping PCR.

To confirm that $\mathrm{P}_{D D I 2}$ could switch on/off gene expression on the yeast genome, we labeled the two target genes $(R A D 18$ and TUP1) with $5 \times$ Flag tag in both the promoter-shuffled and corresponding wild-type strains. In order to determine the relationship between protein abundance and cyanamide concentrations, yFYV12 ( $\mathrm{P}_{D D I 2}-\mathrm{RAD18}$ ) cells were induced with $0-9 \mathrm{mM}$ cyanamide (Fig. 3a). It can be seen that the abundance of the Rad18-5 $\times$ FLAG increased linearly after the 0-9 mM cyanamide induction and almost no signal is detected in the absence of cyanamide. The subsequent quantitative analyses show that protein abundance is linearly related to the cyanamide concentration (Fig. 3a). Hence, the protein abundance can be quantitatively regulated by the $\mathrm{P}_{D D I 2}$.

Because rad18 $\Delta$ strain is radiation sensitive, we measured the survival rate of cells after irradiation with different UV doses. The yFYV12 (0/8 mM), yFYV15 (rad18 $\Delta$ ) and wild-type cells were respectively spread on YPD plates containing cyanamide, then exposed with different doses of UV. The yFYV12 ( $\left.\mathrm{P}_{D D I 2}-R A D 18\right)$ cells that were treated with 0 and $8 \mathrm{mM}$ cyanamide show similar survival rates as yFYV15 (rad18 $\Delta$ ) and wild-type strains, respectively (Fig. 3b).

For the yFYV13 ( $\left.\mathrm{P}_{D D I 2}-T U P 1\right)$ cells, we first observed phenotypic changes at different cyanamide concentrations. The tup $1 \Delta$ strain (yFYV16) was used as a negative control. As shown in Fig. 3c, the yFYV13 cells without cyanamide settle on the bottom of the tube, similar to the yFYV16 strain. Meanwhile, yFYV13 cells induced 
a
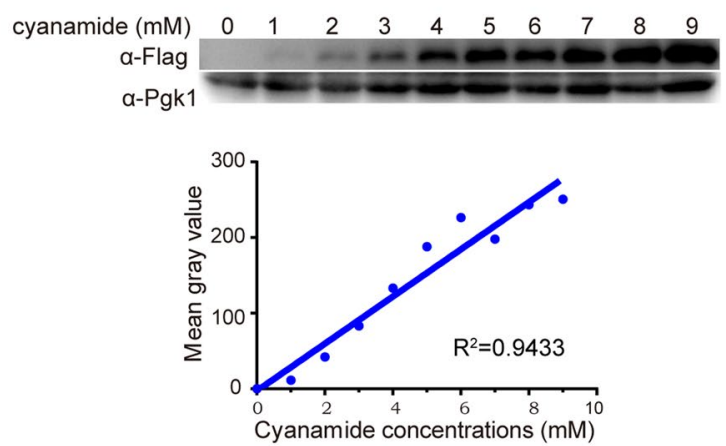

C

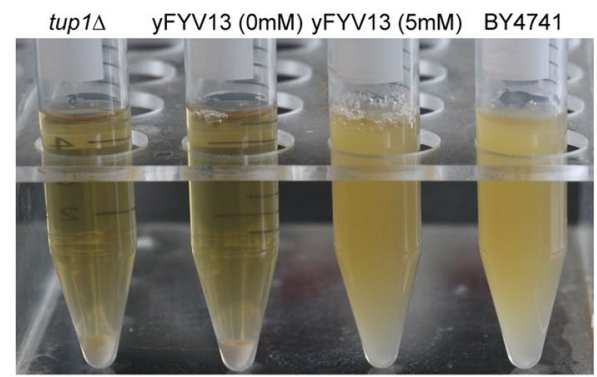

b

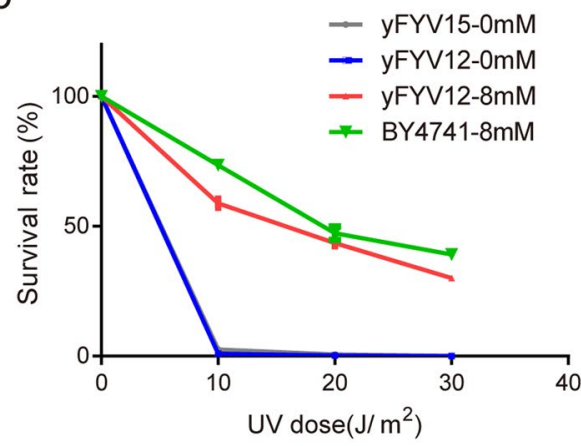

d

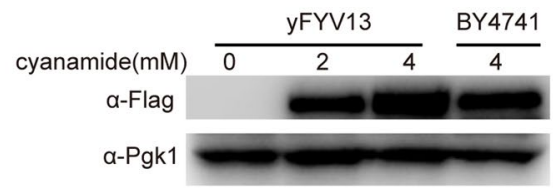

Figure 3. Switching the target gene promoters with the DDI2 promoter in vivo. (a) yFYV12 $\left(\mathrm{P}_{D D I 2^{-}}\right.$ RAD18-5 $\times$ FLAG) cells were induced with 0-9 $\mathrm{mM}$ cyanamide for $4 \mathrm{~h}$, then the total protein was extracted. The abundance of the Rad18 protein was measured by western blot analysis using an anti-Flag antibody. The Pgk1 protein was used as an internal loading control. The mean gray value of Rad18 in the western blot was quantified using Image J software (1.50i). The image of blots is cut from the image of Full-length blots, which are presented in Supplementary Fig. S2. The linear equation $(y=30.16 x-2.67)$ fitted to the change of gray values. (b) Survival rates after UV treatment of strains BY4741, yFYV15 (rad18 $\Delta)$, and yFYV12 $\left(\mathrm{P}_{D D 12}-R A D 18\right)$ with or without cyanamide. (c) yFYV16 (tup1 $\Delta)$, BY4741, and yFYV13 $\left(\mathrm{P}_{D D I 2^{-}}\right.$TUP1) cells were incubated overnight with 0 or $5 \mathrm{mM}$ cyanamide, and then the cultures were imaged. (d) yFYV13 ( $\left.\mathrm{P}_{\text {DDI2 }}-T U P 1\right)$ cells were induced by cyanamide $(0,2$, or $4 \mathrm{mM})$ for $4 \mathrm{~h}$, then the total protein was extracted from yFYV13 and BY4741. Tup 1 protein abundance was measured by western blot analysis using an anti-Flag antibody. The image of blots is cut from the image of Full-length blots, which are presented in Supplementary Fig. S3.

with $5 \mathrm{mM}$ cyanamide were suspended in the same medium as the wild-type cells. Next, we examined the Tup 1-5 $\times$ FLAG protein abundance by Western blot after treatment with 0,2 , and $4 \mathrm{mM}$ cyanamide, respectively. We detected an obvious increase in Tup 1-5 $\times$ FLAG protein level after cyanamide treatment ( 2 or $4 \mathrm{mM}$ ), and no Tup1-5 $\times$ FLAG protein expression was detected without inducer cyanamide (Fig. $3 \mathrm{~d}$ ). The results indicate that, by using different concentrations of cyanamide, $\mathrm{P}_{D D I 2}$ is capable of boosting the expression of the target gene to a level that compares to the tup $1 \Delta$ and wild-type strains. Together, these data suggest that the $\mathrm{P}_{D D I 2}$ gene switch can fine-tune the expression of the selected target genes.

Using DDI2 promoter controls the expression of essential gene CDC6. To investigate whether the $\mathrm{P}_{D D I 2}$ can linearly and tightly regulate the expression of essential genes as well. Here, we chose CDC6 encoding the protein required in the pre-replicative complex formation in DNA replication ${ }^{44}$ as a gene of interest. Compared to other essential gene manipulating method which must transfer the strain with a wild copy of the essential gene to ensure the survival during the modification, we replaced the promoter of CDC6 with the $\mathrm{P}_{D D I 2}$ in a simple and efficient method. We designed a $\mathrm{P}_{D D I 2}$-down fragment (Fig. 4a) which partially drove the expression of the essential gene under the induction of cyanamide. Thus, only adding cyanamide can ensure the cell viability during the promoter replacing process, and an extra wild-type copy of the target gene is unnecessary. Based on the recyclable cassette method ${ }^{37}$, we replaced the CDC6 native promoter with $\mathrm{P}_{D D I 2}$ (Fig. $4 \mathrm{a}$ ).

To confirm that the $\mathrm{P}_{D D I 2}$ can tightly control the essential gene (CDC6) expression as a gene switch. Overnight cultured yFYV14 ( $\left.\mathrm{P}_{D D I 2}-C D C 6\right)$ cells (with $5 \mathrm{mM}$ cyanamide) were respectively transferred into $5 \mathrm{mM}$ cyanamide or cyanamide-free medium for $4 \mathrm{~h}$. Finally, 10000, 1000, and 500 cells were spread on YPD plates containing 0 and $5 \mathrm{mM}$ cyanamide. As shown in Fig. 4c, no cells survive on YPD plates without cyanamide, which clearly indicates that the $\mathrm{P}_{D D I 2}$ is a tight promoter with no detectable expression leakage. The expression of the Cdc6-5 $\times$ FLAG protein was also examined by western blot analysis using an anti-Flag antibody, and a corresponding experimental result is shown in Fig. 4b. It clearly indicates that the protein production corresponds well with the cyanamide concentration (0-5 mM). A small amount of Cdc6-5 $\times$ FLAG protein was detected in $0 \mathrm{mM}$ cyanamide sample probably due to intracellular residual cyanamide from the overnight culture. The western blot results show that a 


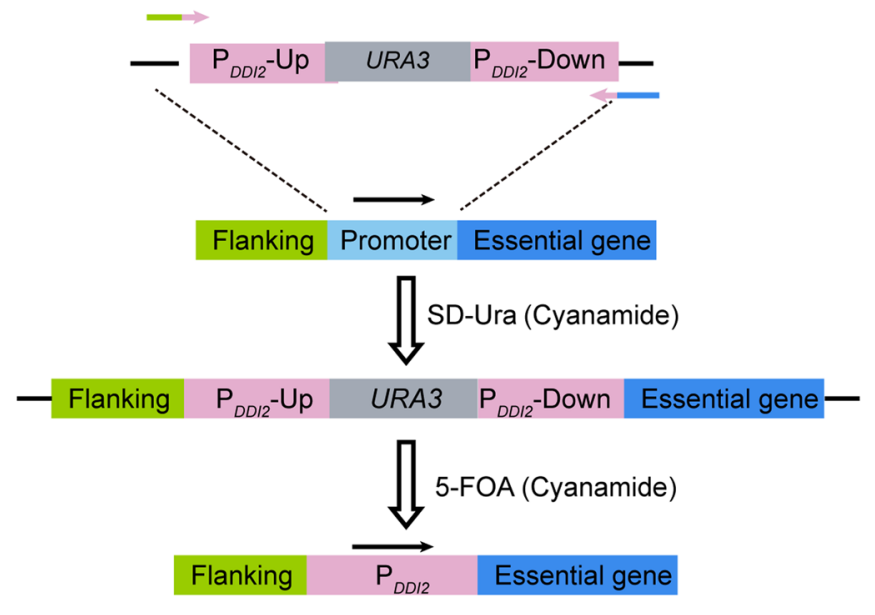

b

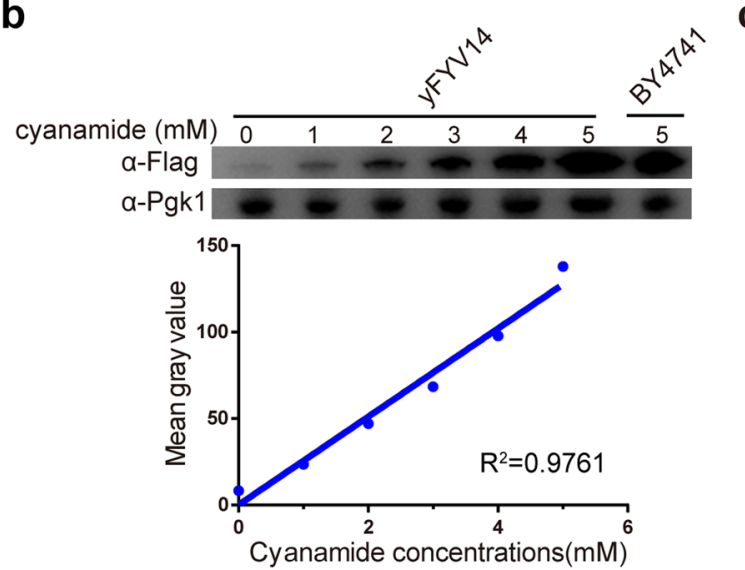

C

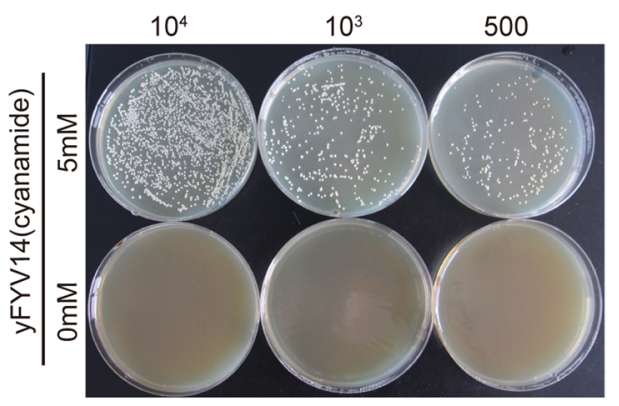

Figure 4. The DDI2 promoter controls the expression of the essential gene CDC6 as a safeguard switch. (a) Schematic illustration of yeast strain construction replacing the essential gene promoter with $\mathrm{P}_{D D I 2}$. The $\mathrm{P}_{D D I 2^{-}}$ $U R A 3-\mathrm{P}_{D D I 2}$ cassette was integrated to replace the native promoter of the essential gene and selected by growing on SD-Ura plates containing cyanamide. Then homologous recombination between these two tandem repeats $\left(\mathrm{P}_{D D I 2}\right)$ results in the pop-out of URA3 along with one copy of the $\mathrm{P}_{D D I 2}$. The addition of the cyanamide can ensure cell survival during the replacement of the essential gene promoter. (b) yFYV14 ( $\mathrm{P}_{D D I 2}$-CDC6) cells were induced by $0-5 \mathrm{mM}$ cyanamide for $4 \mathrm{~h}$. Total protein extracts of yFYV14 and BY4741 were measured by western blot analysis using an anti-Flag antibody. The image of blots is cut from the image of Full-length blots, which are presented in Supplementary Fig. S4. The linear equation $(y=25.49 x+0.13)$ fitted to the change of gray values. (c) The yFYV14 strain was induced with 0 or $5 \mathrm{mM}$ cyanamide. Then, a total of $10^{4}, 10^{3}$, and 500 cells of yFYV14 were plated on YPD or cyanamide-containing YPD $(5 \mathrm{mM})$ plates and incubated at $30^{\circ} \mathrm{C}$ for 3 days.

linear equation fitted the changes in protein abundance. Together, these results implied that the $\mathrm{P}_{D D I 2}$ can act as a gene switch to control the expression of essential genes.

\section{Discussion}

In this study, we demonstrate that $\mathrm{P}_{D D I 2}$ is a strong inducible promoter. We establish a simple promoter switching method to control the expression of target genes on its genomic locus in budding yeast and quantitatively analyzed the expression of target genes under the control of $\mathrm{P}_{D D I 2}$. Our study suggests the potential of $\mathrm{P}_{D D I 2}$ for linear control of gene expression and as a tight on-off switch.

Four plasmids, containing constitutive promoter $A D H 1$ or inducible promoters GAL1, CUP1 and DDI2 linked to the $s f G F P$ reporter gene were constructed to compare promoter strengths. The experimental results show that the strength of $\mathrm{P}_{D D I 2}$ is stronger than all $\mathrm{P}_{A D H 1}, \mathrm{P}_{C U P 1}$, and $\mathrm{P}_{G A L 1}$ (Fig. 2). It has been reported that inducible promoter $\mathrm{P}_{C U P 1}$ is leaky ${ }^{45,46}$. We also observed a rather high basal expressing level of $s f G F P$ even in absence of $\mathrm{CuSO}_{4}$ induction (data not shown). Both GAL1 and DDI2 are stringent promoters. In our experiments, the sfGFP expression under control of these two promoters was undetectable in the absence of corresponding inducers, which is important in cases where background expression is unacceptable, such as the expression of proteins is toxic to yeast cell. Unlike $\mathrm{P}_{G A L 1}, \mathrm{P}_{D D I 2}$ did not require replacement of the culture medium during the process 


\begin{tabular}{|c|c|}
\hline Strains & Genotype \\
\hline W303 & MATa leu2-3,112 trp1-1 can1-100 ura3-1 ade2-1 his3-11,15 \\
\hline BY4741 & MATa his $3 \Delta 1$ leu $2 \Delta 0$ met $15 \Delta 0$ ura $3 \Delta 0$ \\
\hline yFYV9 & W303 with YCplac111- $\mathrm{P}_{A D H 1}-S f G F P-H I S_{6}-\mathrm{T}_{C Y C 1}$ \\
\hline yFYV10 & W303 with YCplac111-P $\mathrm{P}_{G A L 1}-s f G F P-H I S_{6}-\mathrm{T}_{C Y C 1}$ \\
\hline yFYV11 & W303 with YCplac111- $\mathrm{P}_{D D I 2}-s f G F P-H I S_{6}-\mathrm{T}_{C Y C I}$ \\
\hline yFYV12 & BY4741 $\mathrm{P}_{D D I^{2}}-R A D 18-5 \times$ FLAG::natMX6 \\
\hline yFYV13 & BY4741 $\mathrm{P}_{D D I 2}-T U P 1-5 \times F L A G::$ natMX6 \\
\hline yFYV14 & BY4741 $\mathrm{P}_{D D I 2^{-}}{ }^{-C D C 6-5} \times$ FLAG::natMX6 \\
\hline yFYV15 & BY4741 rad18:: natMX6 \\
\hline yFYV16 & BY4741 tup1:: natMX6 \\
\hline yFYV17 & W303 with YCplac111-P $\mathrm{P}_{C U P 1}-S f G F P-H I S_{6}-\mathrm{T}_{C Y C 1}$ \\
\hline
\end{tabular}

Table 1. Yeast strains used in this study.

of induction, thus total culture time was shorter. Moreover, the induced strength of $\mathrm{P}_{D D I 2}$ was higher than $\mathrm{P}_{\text {GAL1 }}$ under the corresponding conditions, and the cost of the cyanamide inducer was less than that of $\mathrm{P}_{G A L 1}$ (galactose $)^{8}$. Accordingly, using $\mathrm{P}_{D D I 2}$ to precisely control gene expression can save a lot of effort and be economical for researchers. More importantly, due to the low price of cyanamide, it has good application prospects in the industrial production that requires high-level induction of target gene with low cost.

Furthermore, the DDI2 promoter can act as a linear gene switch to regulate the gene expression. As a proof-of-concept, the parental promoters of RAD18, TUP1, CDC6 genes were successfully replaced with DDI2 promoter in the genomic locus, so that the expression of the above three genes are under the control of $\mathrm{P}_{D D I 2}$ as desired. Especially, for regulating an essential gene on its genomic locus, there are several advantages for the $\mathrm{P}_{D D I 2}$-shuffling method over other gene regulated expression systems that function in yeast. Because of the special character of DDI2 promoter, an extra wild-type copy of the target gene is not required to transfer into the host cell during the promoter switching process, which is simply not possible with other systems. Thus, the counter-selection step for losing the extra copy of the target gene can be omitted to save time and the cost. The linear correlation between cyanamide concentration and expression level is a great benefit for regulating an essential gene, the target essential gene would be expected to produce the designed amount of product to carry out its function.

Recently, the booming development of synthetic biology has made genetically engineered budding yeast strains more widely used in large-scale cultivation and open environments. To prevent unconscious diffusion of genetically engineered strains into natural ecosystems or protect the intellectual property, an important strategy is utilizing on-off switch that strictly controls the expression of essential genes ${ }^{47}$. According to our data, we anticipate that $\mathrm{P}_{D D I 2}$ can be used as such a switch. In our study, the parental promoter of the essential gene CDC6 was successfully replaced by $\mathrm{P}_{D D I 2}$ in the genomic locus of $S$. cerevisiae. $\mathrm{P}_{D D I 2}$ tight regulated CDC6 expression and the yeast cells did not survive without the induction of cyanamide. In the future, we anticipate $\mathrm{P}_{D D I 2}$ system could be used as a safeguard switch in the recombinant yeast to achieve the integral biosafety.

We envision that DDI2 promoter is used for fine-tuning the expression of the interested gene in both the experimental studies and industrial applications. The main concern for the widespread use of DDI2 promoter could be the low toxicity of inducer cyanamide. The cyanamide can highly induce the expression of DDI2/3 as MMS which is the DNA-damaging agent. It has been reported that cyanamide has modest toxicity to human ${ }^{34}$, however, no direct evidence has been proved that the toxicity is related to DNA damage. Little is known about the toxicity of cyanamide to yeast, although it has been reported that budding yeast cells lacking the cyanamide hydratase activity displayed an enhanced sensitivity to cyanamide ${ }^{24}$. With the development of chemical synthesis technology, it is expected to design the analogs of cyanamide. Hopefully, the analogous compounds can achieve high-level induction as cyanamide, but with non-toxic to cells.

\section{Methods}

Plasmid construction. To compare the strength of four promoters, PCR amplified fragment sfGFP-HIS $6^{-}$ $T_{C Y C 1}$ was inserted into vector YCplac111 between EcoRI and SphI to form YCpLac111-sfGFP-HIS ${ }_{6}-T_{C Y C 1}$. Then, copies of $\mathrm{P}_{A D H 1}, \mathrm{P}_{G A L 1}, \mathrm{P}_{C U P 1}$, and $\mathrm{P}_{D D I 2}$ were cloned into the BamHI and SphI sites of the plasmid YCplac111$s f G F P-H I S_{6}-T_{C Y C 1}$ to form four plasmids: YCplac111- $\mathrm{P}_{A D H 1}-s f G F P-H I S_{6}-\mathrm{T}_{C Y C 1}$, YCplac111- $\mathrm{P}_{G A L 1}-s f G F P-H I S_{6}-$ $\mathrm{T}_{C Y C 1}$, YCplac111-P $\mathrm{P}_{C U P 1}-s f G F P-H I S_{6}-\mathrm{T}_{C Y C 1}$ and YCplac111- $\mathrm{P}_{D D I 2}-s f G F P-H I S_{6}-\mathrm{T}_{C Y C 1}{ }^{37}$.

To obtain the $\mathrm{P}_{D D I 2}-U R A 3-\mathrm{P}_{D D I 2}$ fragments, we inserted the upstream copy of $\mathrm{P}_{D D I 2}$ into the BamHI and EcoRI sites of pBluescript-URA $3^{37,48}$, then the corresponding downstream $\mathrm{P}_{D D I 2}$ fragment was cloned into the HindIII and SalI sites to generate the DUD plasmid (see Supplementary Fig. S1e). To facilitate PCR amplification of the entire DUD cassette, the $3^{\prime}$ end of the upstream promoter and the $5^{\prime}$ end of the downstream copy were truncated to serve as optimized templates.

Strain construction. The yeast strains used in this study are listed in Table 1. Four plasmids with promoter-sfGFP fragments were transformed into yeast strain W303 using a highly efficient LiAc transformation method $^{49}$. The strains were selected on SD-Leu medium (synthetic dextrose medium without leucine) and named yFYV9 $\left(\mathrm{P}_{A D H 1}-s f G F P\right)$, yFYV10 ( $\left.\mathrm{P}_{G A L 1}-s f G F P\right)$, yFYV11 ( $\left.\mathrm{P}_{D D I 2}-s f G F P\right)$ and yFYV17 ( $\left.\mathrm{P}_{C U P 1}-s f G F P\right)($ Table 1$)$. 
The reported recyclable cassettes method ${ }^{37}$ was used to replace the native promoters. The promoters of TUP1, $R A D 18$, and CDC6 yeast genes were scarlessly replaced by $\mathrm{P}_{D D I 2}$, and the resultant strains were named yFYV12, yFYV13, and yFYV14, respectively (Table 1). DUD recyclable cassettes were PCR amplified using target gene promoter-specific primers linked to the cassette-specific primer sequences ${ }^{50}$ and DUD plasmid as template. The primers used in this study are listed in Supplementary Table S1. A two-step method was used to replace the genomic promoter, and the transformed strain was selected on SD-Ura medium. Subsequently, the URA3 marker pop-out strain was achieved by using 5 -FOA based method ${ }^{37}$. The resulting strains were confirmed by genomic PCR followed by Sanger sequencing, and the $5 \times F L A G$ tag was added to the C-terminus of the target genes (TUP1, RAD18, and CDC6) for western blot detection. The RAD18 and TUP1 genes were knocked out using the NAT1 gene, respectively, and the resultant strain was named yFYV15 and yFYV16.

Fluorescence microscopy. yFYV9 $\left(\mathrm{P}_{A D H 1}-s f G F P\right)$, yFYV10 $\left(\mathrm{P}_{G A L 1}-s f G F P\right)$, yFYV11 ( $\left.\mathrm{P}_{D D I 2^{-}}-s f G F P\right)$ and yFYV17 ( $\left.\mathrm{P}_{C U P 1}-s f G F P\right)$ cells were cultured in liquid SD-Leu medium overnight at $30^{\circ} \mathrm{C}$. Next morning, $500 \mu \mathrm{l}$ of the cultures were transferred to tubes containing $5 \mathrm{ml}$ fresh SD-Leu medium (OD600 of 0.4) and incubated for $3 \mathrm{~h}$ to an OD600 of 1.0 to 1.2. Next, the yFYV9 $\left(\mathrm{P}_{A D H 1}-\mathrm{sfGFP}\right)$ cells were cultured in SD-Leu medium for $3 \mathrm{~h}$, and the yFYV10 $\left(\mathrm{P}_{G A L 1}-s f G F P\right)$ cells were starved in raffinose medium for $3 \mathrm{~h}$, then induced in galactose medium for $3 \mathrm{~h}$. The yFYV11 ( $\left.\mathrm{P}_{D D I}-s f G F P\right)$ cells were cultured in SD-Leu medium with $5 \mathrm{mM}$ cyanamide (C87908-100G, Aldrich) for $3 \mathrm{~h}$ and the yFYV17 $\left(\mathrm{P}_{C U P 1}-s f G F P\right)$ cells were cultured in SD-Leu medium with $0.5 \mathrm{mM} \mathrm{CuSO}_{4}$ $(10008218, A C R)$ for $3 \mathrm{~h}$. At the end of incubation, $1 \mathrm{OD}$ yeast cells were collected by centrifugation at $2400 \mathrm{~g}$ for $2 \mathrm{~min}$, washed once with $1 \mathrm{ml} \mathrm{PBS}$, and resuspended in PBS to final OD600 $=40$. Fluorescence microscopy of sfGFP expression was performed using an Olympus FV1200 optical microscope (IX81) with a $60 \times$ objective lens.

Flow cytometry. Cells were incubated and treated as described above. The yFYV9 $\left(\mathrm{P}_{A D H 1}-s f G F P\right)$ cells were cultured for different time points $(2,3$, and $4 \mathrm{~h})$, the yFYV10 $\left(\mathrm{P}_{G A L 1}-s f G F P\right)$ cells were induced with galactose for different induction time points $(2,3$, and $4 \mathrm{~h})$, the yFYV11 $\left(\mathrm{P}_{D D I 2}-s f G F P\right)$ cells were induced with different concentrations of cyanamide $(0-8 \mathrm{mM})$ for $3-5 \mathrm{~h}$, and the yFYV17 $\left(\mathrm{P}_{C U P 1}-s f G F P\right)$ cells were induced with $0.5 \mathrm{mM} \mathrm{Cu}^{2+}$ for different induction time points $(2,3$, and $4 \mathrm{~h})$. After cell culture, $1 \mathrm{OD}$ cells were collected and resuspended in $1 \mathrm{ml}$ PBS. The fluorescence expression of $s f G F P$ in each of the strains was measured using a MoFlo XDP flow cytometry (Beckman). For each strain, approximate 50,000 events were collected to measure fluorescence intensity. Summit 5.2 software was used to analyze the data, and mean fluorescence values were calculated in biological triplicates.

Irradiation, clumping assay, and cyanamide induces the expression of the essential gene. Cultures of yFYV12, yFYV15, and BY4741 were grown overnight in YPD liquid medium, then transferred to fresh medium for another $4 \mathrm{~h}$. Next, $0 / 8 \mathrm{mM}$ cyanamide concentrations were added to the yFYV12 cultures. After $4 \mathrm{~h}$, the yeast cells were harvested and diluted to $10^{4} \mathrm{cells} / \mathrm{ml}$. Then, approximately $50 \mu \mathrm{l}$ of the least diluted cultures were spread on YPD or cyanamide-containing $(8 \mathrm{mM})$ YPD plates in triplicate. The plates were treated with different doses of UV irradiation $\left(0,10,20\right.$, and $\left.30 \mathrm{~J} / \mathrm{m}^{2}\right)$ using a UV cross-linking instrument (Analytik Jena's Model CL-1000), then incubated at $30^{\circ} \mathrm{C}$ for at least 4 days.

The yFYV16, BY4741, and yFYV13 cells supplemented with 0 or $5 \mathrm{mM}$ cyanamide were incubated overnight before imaging.

The yFYV 14 cells were cultured in liquid YPD with $5 \mathrm{mM}$ cyanamide at $30^{\circ} \mathrm{C}$ for $12-16 \mathrm{~h}$. Then, the cells were washed and resuspended in YPD or $5 \mathrm{mM}$ cyanamide-containing YPD medium for $4-6 \mathrm{~h}$. After the cultivation, the yFYV14 cells were harvested and diluted to $10^{5}$ cells $/ \mathrm{ml}$. Approximately $10^{4}, 10^{3}$, or 500 cells were spread on YPD plates (with 0 or $5 \mathrm{mM}$ cyanamide) for 3 days.

Total protein was extracted from yFYV12, yFYV13, and yFYV14 cells induced with different cyanamide concentrations for $4 \mathrm{~h}$, separated by SDS-PAGE, then gels were transferred to a polyvinylidene fluoride membrane and analyzed using immunoblotting. The antibodies used in this study were FLAG tag antibody (M20008M, Abmart) and Pgk1 antibody (ab199438, Abcam). Protein quantification was compared under mean gray value and processed by Image J software (1.50i).

Statistical analysis. Data were presented graphically using GraphPad Prism software. Mean values and standard deviations were obtained for data analyzed in the study. The T test was applied to assess whether the among groups was significantly different. The Significance of statistical analysis was established at $\alpha=0.01$.

\section{Data Availability}

All data generated or analyzed in this study are included in this published article and its Supplementary Information files.

\section{References}

1. Lee, T. I. et al. Transcriptional regulatory networks in Saccharomyces cerevisiae. science 298, 799-804 (2002).

2. Gasch, A. P. et al. Genomic expression programs in the response of yeast cells to environmental changes. Molecular biology of the cell 11, 4241-4257 (2000)

3. Causton, H. C. et al. Remodeling of yeast genome expression in response to environmental changes. Molecular biology of the cell 12, 323-337 (2001)

4. DeRisi, J. L., Iyer, V. R. \& Brown, P. O. Exploring the metabolic and genetic control of gene expression on a genomic scale. Science 278, 680-686 (1997).

5. Orphanides, G. \& Reinberg, D. A unified theory of gene expression. Cell 108, 439-451 (2002).

6. Alper, H., Fischer, C., Nevoigt, E. \& Stephanopoulos, G. Tuning genetic control through promoter engineering. Proceedings of the National Academy of Sciences of the United States of America 102, 12678-12683 (2005).

7. Maya, D., Quintero, M. J., de la Cruz Munoz-Centeno, M. \& Chavez, S. Systems for applied gene control in Saccharomyces cerevisiae. Biotechnol Lett 30, 979-987 (2008). 
8. Romanos, M. A., Scorer, C. A. \& Clare, J. J. Foreign gene expression in yeast: a review. Yeast 8, 423-488 (1992).

9. Da Silva, N. A. \& Srikrishnan, S. Introduction and expression of genes for metabolic engineering applications in Saccharomyces cerevisiae. FEMS yeast research 12, 197-214 (2012).

10. Denis, C. L., Ferguson, J. \& Young, E. mRNA levels for the fermentative alcohol dehydrogenase of Saccharomyces cerevisiae decrease upon growth on a nonfermentable carbon source. Journal of Biological Chemistry 258, 1165-1171 (1983).

11. Holland, M. J. \& Holland, J. P. Isolation and identification of yeast messenger ribonucleic acids coding for enolase, glyceraldehyde3-phosphate dehydrogenase, and phosphoglycerate kinase. Biochemistry 17, 4900-4907 (1978).

12. Johnston, M. A model fungal gene regulatory mechanism: the GAL genes of Saccharomyces cerevisiae. Microbiological reviews 51, 458 (1987).

13. Bassel, J. \& Mortimer, R. Genetic order of the galactose structural genes in Saccharomyces cerevisiae. Journal of bacteriology 108, 179-183 (1971)

14. Douglas, H. \& Hawthorne, D. Enzymatic expression and genetic linkage of genes controlling galactose utilization in Saccharomyces. Genetics 49, 837 (1964)

15. Adams, B. G. Induction of galactokinase in Saccharomyces cerevisiae: kinetics of induction and glucose effects. Journal of bacteriology 111, 308-315 (1972).

16. Macreadie, I. G., Horaitis, O., Vaughan, P. R. \& Clark-Walker, G. D. Constitutive expression of the Saccharomyces cerevisiae CUP1 gene in Kluyveromyces lactis. Yeast 7, 127-135 (1991).

17. Butt, T. R. et al. Copper metallothionein of yeast, structure of the gene, and regulation of expression. Proc Natl Acad Sci USA 81, 3332-3336 (1984).

18. Gorman, J. A., Clark, P. E., Lee, M. C., Debouck, C. \& Rosenberg, M. Regulation of the yeast metallothionein gene. Gene 48, 13-22 (1986).

19. Baron, U. \& Bujard, H. Tet repressor-based system for regulated gene expression in eukaryotic cells: principles and advances. Methods Enzymol 327, 401-421 (2000).

20. Berger, S. M. et al. Quantitative analysis of conditional gene inactivation using rationally designed, tetracycline-controlled miRNAs. Nucleic Acids Res 38, e168 (2010).

21. Dingermann, T. et al. RNA polymerase III catalysed transcription can be regulated in Saccharomyces cerevisiae by the bacterial tetracycline repressor-operator system. EMBO J 11, 1487-1492 (1992).

22. Urlinger, S. et al. Exploring the sequence space for tetracycline-dependent transcriptional activators: novel mutations yield expanded range and sensitivity. Proc Natl Acad Sci USA 97, 7963-7968 (2000).

23. Fu, Y. et al. Rad6-Rad18 mediates a eukaryotic SOS response by ubiquitinating the 9-1-1 checkpoint clamp. Cell 133, 601-611 (2008).

24. Li, J. et al. Two duplicated genes DDI2 and DDI3 in budding yeast encode a cyanamide hydratase and are induced by cyanamide. Journal of Biological Chemistry 290, 12664-12675 (2015).

25. Aravind, L. \& Koonin, E. V. The HD domain defines a new superfamily of metal-dependent phosphohydrolases. Trends in biochemical sciences 23, 469-472 (1998).

26. Maier-Greiner, U. H. et al. Isolation and properties of a nitrile hydratase from the soil fungus Myrothecium verrucaria that is highly specific for the fertilizer cyanamide and cloning of its gene. Proceedings of the National Academy of Sciences 88, 4260-4264 (1991).

27. Stransky, H. \& Amberger, A. Isolation and Properties of A Cyanamide Hydratase (Ec-Group4, 2, 1) From Myrothecium-Verrucaria Alb And Schw. Zeitschrift fur Pflanzenphysiologie 70, 74-87 (1973).

28. Lin, A. et al. Utilization of a Strongly Inducible DDI2 Promoter to Control Gene Expression in Saccharomyces cerevisiae. Front Microbiol 9, 2736 (2018).

29. Lee, M. J., Nagasawa, H. T., Elberling, J. A. \& DeMaster, E. G. Prodrugs of nitroxyl as inhibitors of aldehyde dehydrogenase. Journal of medicinal chemistry 35, 3648-3652 (1992).

30. Lopez, B. E. et al. Inhibition of yeast glycolysis by nitroxyl (HNO): A mechanism of HNO toxicity and implications to HNO biology. Archives of biochemistry and biophysics 442, 140-148 (2005).

31. Katla, V. R., Syed, R., Kuruva, C. S., Kuntrapakam, H. K. \& Chamarthi, N. R. Synthesis of novel phosphorylated guanidine derivatives from cyanamide and their anti-inflammatory activity. Chemical and Pharmaceutical Bulletin 61, 25-32 (2013).

32. Pedelacq, J. D., Cabantous, S., Tran, T., Terwilliger, T. C. \& Waldo, G. S. Engineering and characterization of a superfolder green fluorescent protein. Nat Biotechnol 24, 79-88 (2006).

33. Stearns, T., Ma, H. \& Botstein, D. [23] Manipulating yeast genome using plasmid vectors. Methods in enzymology 185, 280-297 (1990).

34. Schep, L., Temple, W. \& Beasley, M. The adverse effects of hydrogen cyanamide on human health: an evaluation of inquiries to the New Zealand National Poisons Centre. Clin Toxicol (Phila) 47, 58-60 (2009).

35. Douglas, P. M. et al. Chaperone-dependent amyloid assembly protects cells from prion toxicity. Proc Natl Acad Sci USA 105, 7206-7211 (2008).

36. Mann, R. K. \& Grunstein, M. Histone H3 N-terminal mutations allow hyperactivation of the yeast GAL1 gene in vivo. The EMBO journal 11, 3297 (1992).

37. Tian, X., Xu, X. \& Xiao, W. Novel method for genomic promoter shuffling by using recyclable cassettes. Applied and environmental microbiology 79, 7042-7047 (2013).

38. Longtine, M. S. et al. Additional modules for versatile and economical PCR-based gene deletion and modification in Saccharomyces cerevisiae. Yeast 14, 953-961 (1998).

39. Kristoffersen, P., Jensen, G., Gerdes, K. \& Piškur, J. Bacterial toxin-antitoxin gene system as containment control in yeast cells Applied and environmental microbiology 66, 5524-5526 (2000).

40. Bailly, V., Lauder, S., Prakash, S. \& Prakash, L. Yeast DNA Repair Proteins Rad6 and Rad18 Form a Heterodimer That Has Ubiquitin Conjugating, DNA Binding, and ATP Hydrolytic Activities. Journal of Biological Chemistry 272, 23360-23365 (1997).

41. Keleher, C. A., Redd, M. J. \& Schultz, J. Marian Carlson, Johnson A. D. SsnG-Tupl Is a General Repressor of Transbription in Yeast. Cell 68, 709-719 (1992).

42. Wu, J., Suka, N., Carlson, M. \& Grunstein, M. TUP1 Utilizes Histone H3/H2B-Specific HDA1 Deacetylase to Repress Gene Activity in Yeast. Molecular Cell 7, 117-126 (2001).

43. Landgraf, D., Huh, D., Hallacli, E. \& Lindquist, S. Scarless Gene Tagging with One-Step Transformation and Two-Step Selection in Saccharomyces cerevisiae and Schizosaccharomyces pombe. PLoS One 11, e0163950 (2016).

44. Xue, H., Xu, X. \& Fu, Y. V. New insights in pre-replication complex formation with single-molecule visualization. Science Bulletin 60 , $1133-1135(2015)$

45. Peng, B., Williams, T. C., Henry, M., Nielsen, L. K. \& Vickers, C. E. Controlling heterologous gene expression in yeast cell factories on different carbon substrates and across the diauxic shift: a comparison of yeast promoter activities. Microb Cell Fact 14,91 (2015).

46. Lee, K. M. \& DaSilva, N. A. Evaluation of the Saccharomyces cerevisiae ADH2 promoter for protein synthesis. Yeast 22, 431-440 (2005).

47. Cai, Y. et al. Intrinsic biocontainment: multiplex genome safeguards combine transcriptional and recombinational control of essential yeast genes. Proceedings of the National Academy of Sciences 112, 1803-1808 (2015).

48. Wang, Q. et al. A method for labeling proteins with tags at the native genomic loci in budding yeast. PloS one 12, e0176184 (2017). 
49. Ito, H., Fukuda, Y., Murata, K. \& kimura, A. Transformation of Intact Yeast Cells Treated with Alkali Cations. Journal Of Bacteriology 153, 163-168 (1983).

50. Güldener, U., Heck, S., Fiedler, T., Beinhauer, J. \& Hegemann, J. H. A new efficient gene disruption cassette for repeated use in budding yeast. Nucleic Acids Research 24, 2519-2524 (1996).

\section{Acknowledgements}

We thank Dr. Wei Xiao, Xiuqiang Chen for helpful discussions, Dr. Margaret Biswas for English text editing, and the members of our laboratory for valuable feedback on the manuscript. This research was supported by the National Natural Science Foundation of China (31571288), the Newton Advanced Fellowship (NA140085) from the Royal Society, and CAS Interdisciplinary Innovation Team.

\section{Author Contributions}

Y.W., K.Z., X.X., P.W. and Y.F. designed the experiments, Y.W., K.Z. and H.L. performed the experiments, Y.W., K.Z., H.L., X.X., H.X., P.W. and Y.F. analyzed the data, Y.W., K.Z. H.L., P.W. and Y.F. prepared the manuscript, Funding was granted to Y.F.

\section{Additional Information}

Supplementary information accompanies this paper at https://doi.org/10.1038/s41598-019-49000-8.

Competing Interests: The authors declare no competing interests.

Publisher's note: Springer Nature remains neutral with regard to jurisdictional claims in published maps and institutional affiliations.

(c) (i) Open Access This article is licensed under a Creative Commons Attribution 4.0 International License, which permits use, sharing, adaptation, distribution and reproduction in any medium or format, as long as you give appropriate credit to the original author(s) and the source, provide a link to the Creative Commons license, and indicate if changes were made. The images or other third party material in this article are included in the article's Creative Commons license, unless indicated otherwise in a credit line to the material. If material is not included in the article's Creative Commons license and your intended use is not permitted by statutory regulation or exceeds the permitted use, you will need to obtain permission directly from the copyright holder. To view a copy of this license, visit http://creativecommons.org/licenses/by/4.0/.

(C) The Author(s) 2019 